\title{
Simulation of Atomic Force Microscopy Images of Collagen Molecules on Graphite Surface *
}

\author{
Katsunori Tagami ${ }^{\dagger}$ and Masaru Tsukada \\ Department of Nano-Science and Nano-engineering, \\ School of Science and Engineering, Waseda University, \\ 513 Wasedatsurumaki-cho, Shinjuku-ku, Tokyo 162-0041, Japan \\ (Received 6 November 2005; Accepted 18 February 2006; Published 26 March 2006)

\begin{abstract}
Based on the all-atom empirical force field model, we investigated the force distance curves and the corresponding atomic force microscopy (AFM) images for the single collagen molecule adsorbed on the graphite surface, both in UHV and in water, assuming the $(10,0)$ capped carbon nanotube tip. We found that in UHV the height difference between the proline and glycine amino residues can be clearly resolved in the images, whether the molecule assumes the triple helix structure or its deformed structure which is energetically more favorable. In water, the amplitude of the oscillatory solvation force is found to be different between on top of the collagen molecule and on top of the graphite surface. It indicates that the collagen molecule would be observed to be lower in water than the molecule observed in UHV environment. [DOI: 10.1380/ejssnt.2006.294]
\end{abstract}

Keywords: Bioimaging and engineering; Biological molecules - proteins; Atomic force microscopy; Collagen; Solvation force; Water; Computer simulations; Semi-empirical models and model calculations

\section{INTRODUCTION}

Recently, atomic force microscopy (AFM) has been widely used to investigate the mechanical properties such as force distance curves as well as their topographic images for various biomolecules. In most cases, the biomolecules are immobilized by being adsorbed on substrates or packed in the 2-dimensional lattice [1-6]. Such an improvement on the experimental techniques has led to subnanometer resolved images on lateral directions [7]. However, the biomolecules easily change their structure due to their surrounding environments, i.e., adsorption on the substrate, temperature, humidity, and so on. This makes it difficult to analyze what is actually observed in experimentally obtained images and what is the origin of the force distance curves. Thus, theoretical studies based on simulations are getting important for understanding the detailed mechanism which are behind the observed phenomena in experiments.

Our purpose in this work is to develop a method of theoretical simulations of AFM images of biomolecules adsorbed on substrates under various conditions. In this article, we choose a case study of a single collagen molecule (fragment) adsorbed on the graphite surface, and examine the developed simulation method both under dry (ultra high vacuum, UHV) and wet (in water) conditions. The collagen molecules themselves have been extensively studied by AFM, and clear images have been observed in many experiments [8-17]. However, these collagen molecules take the bundled structure like fibril, whose sizes are much larger than a single collagen molecule. The AFM images for the latter have been reported in the literature [18-20] but with low resolutions, and not have not been studied intensively. As for the tip, it is modeled as the capped

*This paper was presented at International Symposium on Surface Science and Nanotechnology (ISSS-4), Saitama, Japan, 14-17 November, 2005.

$\dagger$ Corresponding author: tagami@cms.nano.waseda.ac.jp carbon nanotube which has an advantage in experiments [21-24] due to their definite structure and high aspect ratio. The force distance curves are calculated using the program called NAMD 2.5 [25] with the all-atom empirical force field called $C H A R M M$.

We found the following characteristic features by the simulation; (1) In UHV, the single collagen molecule adsorbed on the graphite surface can easily deform its structure due to its thermal motion. However, provided that the molecules can be immobilized on the surface, the height difference between the proline (PRO) and glycine (GLY) amino residues can be clearly resolved in the images, whether the molecule assumes the triple helix structure or its deformed structure. (2) In water, the collagen molecule can retain the triple helix structure. The amplitude of the oscillatory solvation force due to the quasilayer structure of water near the interface is different between on top of the collagen and on top of the graphite surface. It indicates that the collagen molecule would be observed to be lower in the image in water than the collagen observed in UHV environment.

The rest of the paper is organized as follows. In $\S I I$ the computational model and simulation method will be described. In $\S I I I$ the results of our calculations will be presented and the major characteristics of the simulated images will be discussed. In $\S \mathrm{IV}$ the paper will be summarized.

\section{MODEL AND METHOD}

We assume the supercell structure whose size is 68.28 $\times 157.7 \times 100 \AA^{3}$. At the bottom of each supercell, a graphite sheet of monolayer thickness is located, and a single collagen fragment is deposited on this substrate. The lateral dimensions of the graphite substrate are assumed to be $68.28 \times 157.7 \AA^{2}$. The collagen fragment is assumed to take a triple helix structure each of whose strand is composed of only 12 GLY and 24 PRO residues, i.e., (GLY-PRO-PRO) 12 [26]. Before the structural opti- 

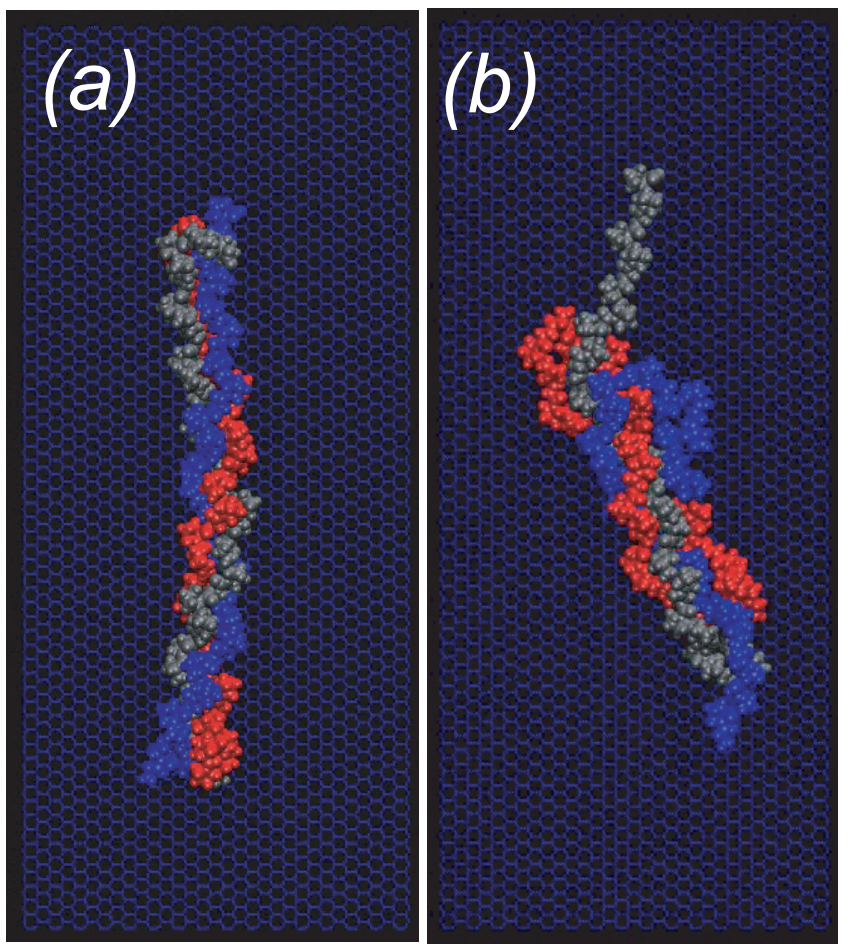

FIG. 1: Top view of optimized collagen adsorbed on graphite substrate. (a) the triple helix structure, (b) its deformed structure. The lateral dimensions of the substrate shown are 68.28 $\times 157.7 \AA^{2}$.

mization, its diameter and length are about $15 \sim 20 \AA$ and 107.6 $\AA$, respectively. The water molecules are added so that the vacuum/water interface lies at around $30 \AA$ from the surface, by which the collagen molecule are immersed completely. The AFM tip is modeled by the $(10,0)$ carbon nanotube with both ends capped. The diameter and length of the tip are about $8 \AA$ and $37.5 \AA$, respectively. The axis of the tip is set to be perpendicular to the graphite surface.

The number of atoms in each component is 1,269 (collagen), 4,096 (graphite), 26,295 (water), and 360 (nanotube), respectively, which amounts to 32,020 atoms in total. The interatomic interaction is treated by the all-atom empirical force field called CHARMM. For the graphite and nanotube, we adopt the potential parameter for the atom type "CA". For the water molecules, we adopt the TIP3P potential model. The long-range interaction is calculated by the Particle Mesh Ewald (PME) sum method. The size of the PME grids is assumed to be 32, 64, and 64 for the corresponding directions.

The whole simulations are performed in the following way. In the case without water molecules, which mimics the UHV environment, we consider two types of structures as illustrated in Fig. 1 [27]. Figure 1 (a) corresponds to the structure obtained after the molecular dynamics (MD) simulation at $T=300 \mathrm{~K}$ for $0.3 \mathrm{~ns}$, and Fig. 1 (b) corresponds to the structure obtained after the MD simulation at $T=300 \mathrm{~K}$ for $3.1 \mathrm{~ns}$. Note that these structures are further optimized by the energy minimization method after the corresponding MD simulations. Although the triple helix structure is retained in the former case, it is remarkably destroyed in the latter case. Specifically, in the latter, the upper terminals of the red and blue strands unwind their helix structure, and meet together at around the center of molecule. In addition, the lower terminal of the red strand turns back toward the center. In a sense, the latter configuration is a folded structure of the collagen molecule. Energetically the latter structure is more stable than the former by $573.6 \mathrm{kcal} / \mathrm{mol}$. In both cases, the force distance curves are calculated by scanning the nanotube tip above the collagen/surface assuming that all the components are rigid. The normal and lateral grid spacings of the scan points are set to be 0.1 and $2.0 \AA$, respectively.

In existence of water, the collagen molecule is assumed to take the former configuration obtained above, i.e., the triple helix structure ( Fig. 1(a) ). In fact, the preliminary MD simulations show that the triple helix structure is not destroyed even if they move in water at $T=300$ $K$. The force distance curves are calculated just on two lateral positions, i.e., on top of the collagen molecule and on top of the graphite surface. In each case, first, the rigid nanotube tip is set so that its apex is above the surface of the water. Then, only the water molecules are allowed to move at $T=300 \mathrm{~K}$ for 100,000 steps with the aid of LANGEVIN dynamics method. The time step is assumed to be 2.0 fs with the rigid bond constraints applied on the water molecules. During this MD simulation, the atomic forces inside the tip are monitored. The mean normal force at this tip height is calculated by summing these force components over the whole tip atoms at each MD step, then by averaging during 100,000 MD steps. Next, the tip is moved downward by $0.25 \stackrel{\leftrightarrow}{A}$ with its structure unchanged. Again, the atoms other than the water molecules are fixed. Then, the MD simulation for 100,000 steps is performed. These procedures are performed repeatedly.

\section{RESULTS}

\section{A. Force distance curves in UHV and water}

First, we will present the force distance curves calculated in UHV environment. Figure 2 shows the normal force plotted against the tip height $h$ which is defined to be the distance between the bottom of the nanotube tip and the graphite surface. The red and blue curves correspond to the force distance curves calculated on top of the triple helix collagen molecule ( see Fig. 1(a)) and on top of the graphite surface, respectively. The green curve corresponds to the force distance curve calculated on top of the single strand drawn with the gray color in the upper half of Fig. 1(b). As clearly observed, the tip height at the maximal attractive force is higher in the red curve, $h=17.6 \stackrel{\circ}{A}$, than the corresponding tip height in the green curve, $h=8.5 \AA$. This agrees with the topography of the collagen molecule, i.e., the triple helix is higher than the single strand. In addition, the depths of the attractive forces are significantly different among the three cases, which indicates that the background van der Waals force from the graphite is enhanced upon approaching the substrate surface.

In existence of water, we calculate the force distance curve (a) on top of the collagen molecule and (b) on top 


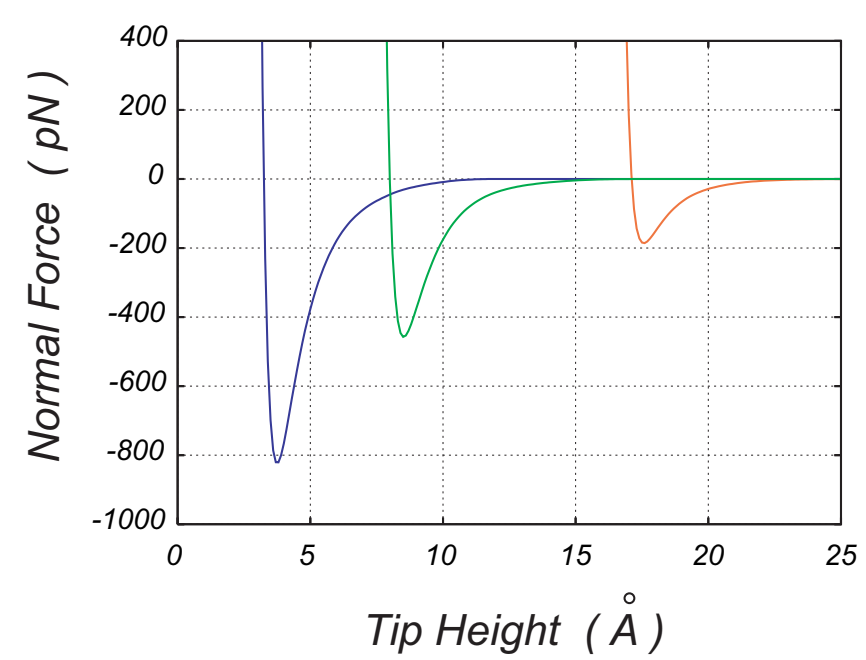

FIG. 2: Force distance curves in UHV. The red and blue curves correspond to the force distance curves on top of the triple strand collagen molecule ( Fig. 1(a) ) and the graphite surface, respectively. The green curve corresponds to the force distance curve on top of the single strand ( Fig. 1(b)).

of the graphite surface, which are shown in Fig. 3. The solid circles denote the mean force values in the normal direction plotted against the tip height $h$. For comparison, the force distance curves calculated without water molecules are plotted by the red curves. In the case (a), at $h>40 \AA$, the tip feels no effective forces, which indicates that the tip is not dipped into water yet. At around $h=35.7 \sim 36.7 \AA$, the tip sometimes interact with the water molecules below it. After this intermittent region, the tip apex is completely wet, and the strength of the attractive force does not change significantly until the tail of the oscillatory peak structures appears at around $h=$ 10.2 $\AA$. The origin of the nearly constant force region at $10.2<h<35.7 \AA$ turns out to be the difference between the capillary force at the side (wall) of the nanotube tip and the hydrostatic force at the tip apex. The former and latter forces work along the downward and upward directions, respectively. It is true that the hydrostatic forces are also formed on the wall of the nanotube, but they cancel out and do not contribute to the normal tip force. In addition, as the diameter of the nanotube tip is constant, the force values do not change significantly once the tip is soaked into water. As the tip is pushed further, the tip feels the oscillation in the force curves whose peaks lies at $h=8.7$ and $5.9 \AA$. The amplitude at the latter peak is found to be much stronger.

This kind of oscillatory force is referred as the solvation force and has been widely investigated in both experiments and theoretical simulations [28-30]. In addition, using the nanotube tip, this quantity has been detected in the recent AFM experiments indirectly as the frequency shift and energy dissipation [23, 24]. The shape of the oscillatory curves has been found to have a relationship with the number of solvation shell between the tip and surface, which highly depends on the nature of the solvent molecule. In particular, in the case of water, the number of solvation shell is known to be about $2 \sim 3$ $[31,32]$, which agrees with the results in our calculations.

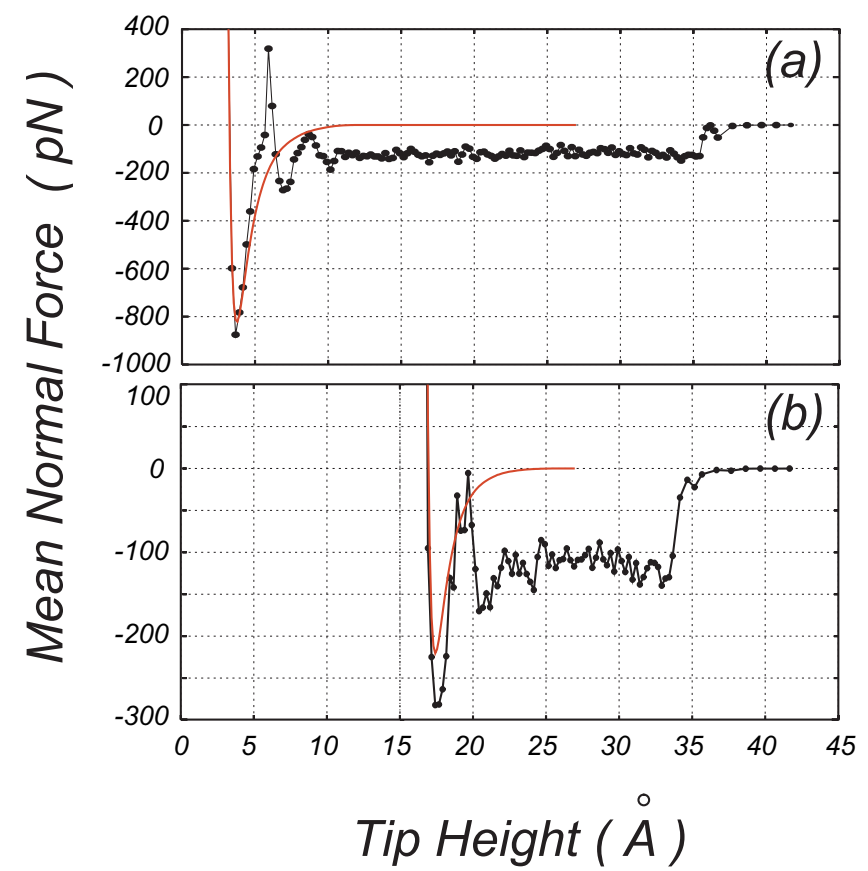

FIG. 3: Force distance curves calculated (a) on top of the graphite surface, and (b) on top of the collagen molecule. The red curves show the force distance curves calculated without water molecules.

On top of the collagen molecule ( case (b) ), a similar trend is observed in the force distance curve. Once the tip apex is completely wet, the tip experiences the comparatively flat normal force region at $24.4<h<34.2$ $\stackrel{\circ}{ }$. The double peak structures are found at around $h=$ 22.2 and $19.7 \AA$, although their shape gets more dull and their peak heights get lower than those observed on top of the graphite surface. It looks as if the number of solvation shell is reduced by one on the collagen molecule compared to that on the flat graphite surface.

\section{B. Simulated AFM images}

The force distance curves as in the previous subsection are calculated with the spacing of $2.0 \AA$ along the lateral directions. Then, the AFM image is obtained by mapping the tip heights which correspond to a certain normal force value on the corresponding tip lateral positions. Figure 4 shows the obtained AFM images in UHV with the constant normal force mode $\left(F_{z}=-25 \mathrm{pN}\right)$ for the molecular configuration in Fig. 1. The image in Fig. 4(a) shows the successive nodal structures, which reflects the triple helix structure of the collagen. To be concrete, the bright areas correspond to the PRO amino residues, whose sizes are larger than those of the GLY residues. In addition, both ends of the collagen molecule are observed to be larger than the central part of the molecule, which agrees with the atomic height difference, i.e., the former is

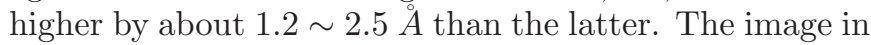
Fig. 4(b) indicates that the AFM can recognize the width difference between the upper and central part of the collagen in the deformed configuration. Furthermore, it is 

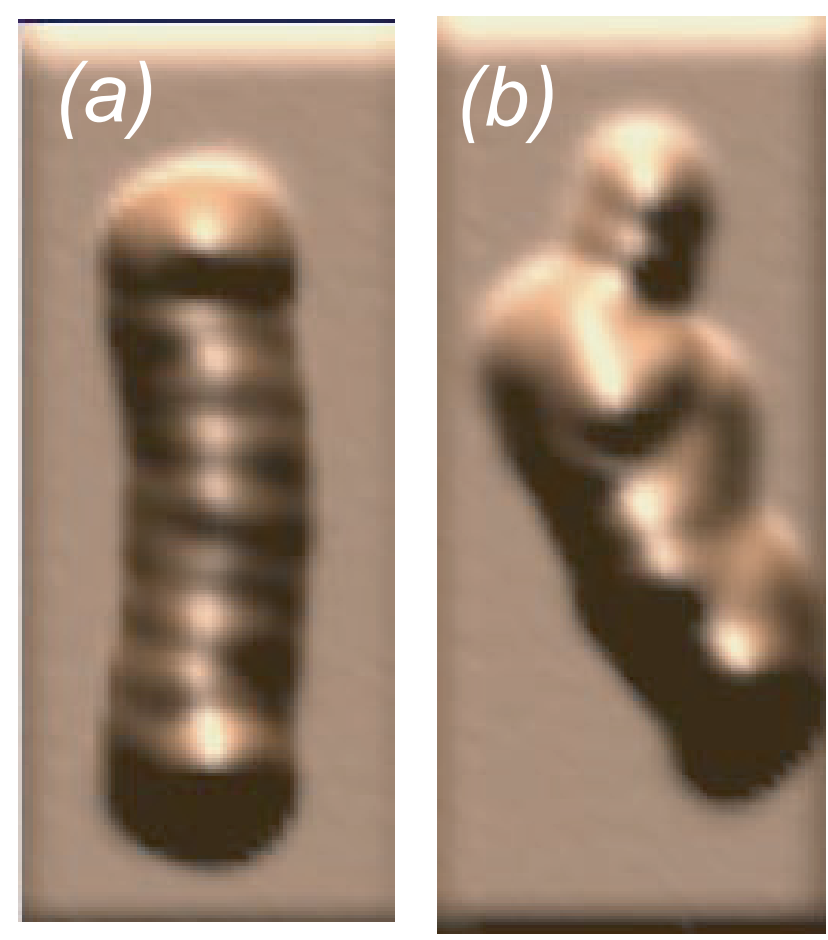

FIG. 4: Simulated AFM images of collagen molecules in UHV for (a) the triple helical structure and (b) its deformed structure. The lateral dimensions of the substrate shown are 68.28 $\times 157.7 \AA^{2}$.

worthy to note that the single strand with the gray color ( see Fig. 1(b) ) is observed to show the nodal structures. The top and bottom of these nodal structures correspond to the PRO and GLY amino residues, respectively, whose height difference is $2.35 \AA$. This indicates that the alignment of the amino residues of any other protein molecules may be recognized using the AFM, if they are unfolded, elongated, and then immobilized on the atomically flat surface, and at the same time if the tip with a sufficiently sharp apex is prepared.

In existence of water, we calculated the force distance curves only on the two lateral positions, and thus we do not obtain the AFM images. However, we can predict the images in water with the aid of the discussion in the previous subsection. In Fig. 3, we found that the difference between the capillary force and the hydrostatic force results in the attractive force, both of which do not depend on the tip height. When the tip is completely immersed in water, which corresponds to the situations in AFM experiments performed in liquid, both contributions diminish and the normal tip force is expected to be zero far from the surface. Namely, the force distance curves are considered to shift upward uniformly by $120 \sim 160 \mathrm{pN}$ compared to Fig. 3. Then, at a certain tip height, the tip first feels the nonzero and oscillatory solvation force. As noted in the previous subsection, the amplitude of the solvation force is different between on top of the collagen molecule and on top of the graphite surface, and the number of solvation shell seems to be reduced by one on the former. It indicates that when the AFM image is generated in the constant normal force mode, the collagen molecule would be observed to be lower by about $2.5 \stackrel{A}{A}$, which is the size of a water molecule, than in the UHV condition. If the tip is pushed further and the water between the tip and molecule/substrate is completely excluded, the tip feels the force directly from the latter. By detecting such force contribution, the AFM images would be expected to be similar to that obtained in UHV, although the strong attractive force may collapse the molecular configuration.

\section{SUMMARY}

Based on the all-atom empirical force field model, we investigated the force distance curves and the corresponding AFM images for the single collagen molecule adsorbed on the graphite surface, both in UHV and in water, assuming the $(10,0)$ capped carbon nanotube tip. We found that in UHV the height difference between the PRO and GLY amino residues can be clearly resolved in the images, whether the molecule assumes the triple helix structure or its deformed structure which is energetically more favorable. In water, the amplitude of the oscillatory solvation force is found to be different between on top of the collagen molecule and on top of the graphite surface. It indicates that the collagen molecule would be observed to be lower in water than the molecule observed in UHV environment.

\section{Acknowledgments}

The authors thank to Dr. H. Sekiguchi and Prof. A. Ikai at Tokyo Institute of Technology for fruitful discussions about AFM observation of biomaterials. This work was supported in part by the Japan Science and Technology Agency (JST) program "Development of System and Technology for Advanced Measurement and Analysis".
[1] A. P. Quist, C. T. Reimann, B. U. R. Sundqvist, L. P. Bjorck, and S. O. Oscarsson, Surf. Sci. 325, 1406 (1995).

[2] F. Valle, G. Dietler, and P. Londei, Biochemical and Biophysical Research Communications 288, 258 (2001).

[3] C. A. Johnson, Y. Yuan, and A. M. Lenhoff, J. Colloid. Interface. Sci. 223, 261 (2000).

[4] D.J. Müller, J.B. Heymann, F. Oesterhelt, C. Möller, H. Gaub, G. Büldt, and A. Engel, Biochimica et Biophysica Acta / Bioenergetics 1460, 27 (2000).
[5] D. J. Müller, H. -J. Sass, S. Müller, G. Büuldt, and A. Engel, J. Mol. Bio. 285, 1903 (1999).

[6] S. Scheuring, P. Ringler, M. Borgina, H. Stahlberg, D.J. Müller, P. Agre, and A. Engel, EMBO J. 18, 4981 (1999).

[7] D. J. Müller, D. Fotiadis, S. Scheuring, S.A. Müller, and A. Engel, Biophys. J. 76, 1101 (1999).

[8] V. Baranauskas, B. C. Vidal, and N. A. Parizotto, Appl. Biochem. Biotechnol. 69, 91 (1998).

[9] M. F. Paige, J. K. Rainey, and M. C. Goh, Biophys. J. 
74, 321 (1998).

[10] D. J. Taatjes, A. S. Quinn, and E. G. Bovill, Microsc. Res. Tech. 44, 347 (1999).

[11] E. Adachi, O. Katsumata, S. Yamashina, D. J. Prockop, and A. Fertala, Matrix. Biol. 18, 189 (1999).

[12] S. Yamamoto, H. Hashizume, J. Hitomi, M. Shigeno, S. Sawaguchi, H. Abe, and T. Ushiki, Arch. Histol. Cytol. 63, 127 (2000) and references therein.

[13] S. Yamamoto, J. Hitomi, S. Sawaguchi, H. Abe, M. Shigeno, and T. Ushiki, Jpn. J. Ophthalmol. 44, 318 (2000).

[14] M. F. Paige and M. C. Goh, Micron 32, 355 (2001).

[15] F. El Feninat, T. H. Ellis, E. Sacher, and I. Stangel, Dent. Mater. 17, 284 (2001).

[16] A. C. Lin and, M. C. Goh, Proteins 49, 378 (2002).

[17] S. Habelitz, M. Balooch, S. J. Marshall, G. Balooch, and G. W. Marshall, J. Struct. Biol. 138, 227 (2002).

[18] M. B. Shattuck, M. G. L. Gustafsson, K. A. Fisher, K. C. Yanagimoto, A. Veis, R. S. Bhatnagar, and J. Clarke, J. Microscopy 174, 1 (1994).

[19] H. Lin, D. O. Clegg, and R. Lal, Biochemistry 38, 9956 (1999).

[20] S. Yamamoto, F. Nakamura, J. Hitomi, M. Shigeno, S. Sawaguchi, H. Abe, and T. Ushiki, J. Electron Microscopy 49423 (2000).

[21] K. I. Hohmura, Y. Itokazu, S. H. Yoshimura, G.
Mizuguchi, Y. Masamura, K. Takeyasu, Y. Shiomi, T. Tsurimoto, H. Nishijima, S. Akita, and Y. Nakayama, J. Electron Microsc. (Tokyo) 49, 415 (2000).

[22] J. Li, A. M. Cassell, and H. Dai, Surf. Interface Anal. 28, 8 (1999).

[23] S. P. Jarvis, T. Uchihashi, T. Ishida, H. Tokumoto, and Y. Nakayama, J. Phys. Chem. B 104, 6091 (2000).

[24] T. Uchihashi, M. Higgins, Y. Nakayama, J. E. Sader, and S. P. Jarvis, Nanotechnology 16, S49 (2005).

[25] L. Kale, R. Skeel, M. Bhandarkar, R. Brunner, A. Gursoy, N. Krawetz, J. Phillips, A. Shinozaki, K. Varadarajan, and K. Schulten, J. Comp. Phys. 151, 282 (1999).

[26] The pdb filename is 1clg.pdb.

[27] The figure is generated using the program VMD. ( see, W. Humphrey, A. Dalke, and K. Schulten, J. Molecular Graphics 14, 33 (1996). )

[28] P. D. Ashby and C. M. Lieber, J. Am. Chem. Soc. 126, 16973 (2004).

[29] J. Gao, W. D. Luedtke, and U. Landman, J. Phys. Chem. B 101, 4013 (1997).

[30] G. Carbone, R. Barberi, I. Musevic, and U. Krzic, Phys. Rev. E 71, 051704 (2005).

[31] R. Qiao and N. R. Aluru, NanoLetters 3, 1013 (2003).

[32] P. Gallo and M. Rovere, J. Phys.: Condens. Matter 15, 1521 (2003). 